\title{
Treatment for Leiomyosarcoma of the Inferior Vena Cava Complicated with Lung Adenocarcinoma: A Case Report and Literature Review
}

Xiaohu Guo ( $\sim$ guoxiaohu2011@126.com )

Lanzhou University Second Hospital

Xiaoyan Liu

Lanzhou University Second Hospital

Zhengang Wei

Lanzhou University Second Hospital

Mancai Wang

Lanzhou University Second Hospital

Youcheng Zhang

Lanzhou University Second Hospital

\section{Case report}

Keywords: Leiomyosarcoma, inferior vena cava, lung adenocarcinoma.

Posted Date: August 4th, 2021

DOl: https://doi.org/10.21203/rs.3.rs-746417/v1

License: (9) This work is licensed under a Creative Commons Attribution 4.0 International License.

Read Full License 


\section{Abstract}

Background: Leiomyosarcoma originating from the inferior vena cava (IVC) is a very rare malignancy. We searched the databases and did not find similar cases of leiomyosarcoma of the IVC with lung adenocarcinoma. Here, we report a patient with IVC leiomyosarcoma and lung adenocarcinoma.

Case report: The patient, a 38-year-old Chinese female, presented to the general department with a history of intermittent right upper abdominal pain for 18 months. Contrast-enhanced computed tomography (CT) showed a tumour of IVC $(3.4 * 2.7 \mathrm{~cm})$ extending to the renal veins. In addition, chest CT showed a tumour in the left lung lobe. Then, the patient underwent surgery to resect the IVC tumour and partial lung. The patient received Gefitinib Tablets treatment $250 \mathrm{mg}$ per day for 18 months during the perioperative period. The examination of the patient showed lung recurrence at the 18th month after partial pneumonectomy. So the patient again received thoracoscopic wedge pneumonectomy, and it was confirmed to be metastasis of IVC tumour. The patient received daily $12 \mathrm{mg}$ treatment with Anlotinib after the last operation. Regular outpatient follow-up showed that there was no obvious recurrence and metastasis as of April 2021 (6 months after operation).

Conclusions: It is difficult to diagnose and treat patients with IVC leiomyosarcoma and lung adenocarcinoma in a timely manner. Surgery is still an effective treatment for patients with IVC leiomyosarcoma and lung adenocarcinoma at present.

\section{Background}

Leiomyosarcoma originating in the inferior vena cava (IVC) is a very rare malignancy, with a few hundred cases reported worldwide [1, 2]. It occurs more commonly in women, with an age of approximately 60 years old [3]. It is difficult to diagnose because of the absence of typical clinical manifestations such as dyspnoea, abdominal pain, distention, back pain, weight loss, and malaise. Primary leiomyosarcoma of the IVC may be under-detected for several years before the diagnosis is made. Because of the lack of early diagnosis and effective treatment, the prognosis of primary leiomyosarcoma of the IVC is very poor, with a median survival of 2 years [4]. Lung cancer has been one of the leading causes of cancer-related death worldwide for two decades [5]. Female patients usually present with lung adenocarcinoma. Leiomyosarcoma of the IVC with lung adenocarcinoma is rare. We searched the databases and did not find similar cases. Here, we report a case of IVC leiomyosarcoma and lung adenocarcinoma.

\section{Case Report}

A 38-year-old female presented to the general department with a history of intermittent right upper abdominal pain, back pain, nausea and vomiting for 18 months. The patient is a college teacher with good living habits and no history of addiction, smoking or family disease. The physical examination was normal. The symptoms did not decrease after the oral administration of an acid-inhibitory drug and cholagogue at the local hospital. 
Ultrasound of the abdomen showed a retroperitoneal tumour $(4.8 * 2.3 \mathrm{~cm})$ (Fig. 1). To further confirm the diagnosis, contrast-enhanced computed tomography (CT) was performed and showed a right retroperitoneal tumour $(3.4 * 2.7 \mathrm{~cm})$ extending to the renal veins and into the partially patent IVC lumen, and it was pushing the pancreas forward. There were no enlarged lymph nodes around the tumour (Fig. 2). To determine the primary site of the tumour and whether metastasis had occurred, we performed head magnetic resonance imaging (MRI), chest CT and cardiac ultrasound. The patient refuses to undergo PET-CT examination in order to save money. The cardiac ultrasound and brain MRI results were normal. The CT scan of the chest showed a tumour in the lingual segment of the upper lobe of the left lung, consisting of ground-glass nodules with unclear boundaries; the tumour was approximately 1.3 $\mathrm{cm} \times 1.1 \mathrm{~cm}$ in size and had multiple short burls on the edges, and there were no enlarged lymph nodes in the mediastinum (Fig. 3). Bronchoscopy was performed to clarify the nature of the tumour, showing acute inflammation of the bronchial mucosa. We considered lung nodules as metastases from the IVC tumour, primary lung cancer or benign lung nodules. Therefore, the patient was discussed in multidisciplinary tumor board for management. The results that Inferior vena cava sarcoma without effective treatment in addition to surgery. We can be conducted first resection of the inferior vena cava sarcoma in our department without preoperative pulmonary biopsy, and then resection of pulmonary tumour by thoracoscope after recovery from the first operation.

A full-length right upper rectus abdominus incision was initially performed. The patient underwent resection of the sarcoma of the inferior vena cava, which was located at the confluence of the renal vein into the inferior vena cava. In order to save operation time, the inferior vena cava was reconstructed with artificial blood vessels. During the surgical procedure, there were no enlarged lymph nodes in the abdominal cavity and no metastatic foci in the abdominal cavity or pelvis. The lateral retroperitoneum of the duodenum was cut, and the duodenum was pulled up to the left. A solid and prominent tumour $(6 * 4 * 3$ $\mathrm{cm}$ ) was located in the middle segment, including the anterolateral wall of the vena cava, as well as the renal veins. Vascular clamps were used to clamp the proximal and distal portions of the IVC tumours, renal arteries and renal veins, and then complete removal of the tumours was performed. Proximal and distal to the IVC, the renal veins were anastomosed with the artificial vessel by $5-0$ Prolene sutures. The vascular clamp was opened to restore blood flow after vascular anastomosis, and no active bleeding was detected. Bleed loss about $400 \mathrm{ml}$ during the operation. There were no obvious complications and without intensive care unit after the operation. The patient was discharged from the hospital on the 13th day after the operation.

The postoperative pathological specimens showed that the tumour was $6 \times 4 \times 3 \mathrm{~cm}$ in size, lobulated, and had an intact capsule, and there was no metastasis in the surrounding lymph nodes. The histopathological examination of the excised mass showed hypercellular mesenchymal tumours that were arranged in fascicles, bundles and interlacing patterns (Fig. 4). The tumour cells were spindleshaped and elongated, with abundant cytoplasm. The nuclei were hyperchromatic, blunt-ended, centrally located and had some degree of atypia and pleomorphism (Fig. 5). A few fused nuclei were also seen. Immunohistochemical staining showed positivity for smooth muscle actin (SMA), myoglobin, desmin, Epithelial Membrane Antigen (EMA), Transducin-Like Enhancer of split 1 (TLE-1) and h-caldesmon; Ki-67 
showed $40 \%$ positivity, and vimentin and CD99 were focally positive; and CD 117, CKp, CK7, Bcl-2, S100, CD31, CD34, Dog-1 and STAT-6 were negative. These findings were suggestive of IVC leiomyosarcoma (Fig. 5).

After one month, the patient underwent thoracoscopic wedge-shaped pneumonectomy. There was a mass less than $1 \mathrm{~cm}$ in diameter in the anterior segment of the left upper lobe after entering the pleural cavity. No obvious pleural contraction or lymph node enlargement was seen in the hilum or mediastinum. The lung tissue, including the tubercle in the left upper lung, was removed more than $2 \mathrm{~cm}$ from the edge of the tubercle with a linear cutting closure device. There were no obvious blood loss and complications during or after the operation. The patient was discharged from the hospital 4 days after thoracoscopic wedge-shaped partial pneumonectomy.

The postoperative pathological specimens showed lung tissue with a size of approximately $5 \times 4 \times 2 \mathrm{~cm}$, and a tumour with a diameter of $0.8 \mathrm{~cm}$ was seen in the centre. The resection margin was larger than 2 $\mathrm{cm}$ from the tumour, and the mediastinal lymph nodes were not dissected. The histopathological examination of the excised mass showed lung adenocarcinoma (Fig. 6). Immunohistochemical staining showed positivity for CK7, napsin A and TTF-1, Ki-67 showed 20\% positivity, and CK5/6, p63, p40, Syn, CgA, CD56 and LCA were negative. CK7, napsin A and TTF-1 were positive in lung adenocarcinoma, while P63 and CK5/ 6 were positive in lung squamous cell carcinoma. Additional, the lung tissue has carried on the consultations to assist in the diagnosis in the cancer hospital of Fudan University and Gansu provincial cancer hospital, and experts from two hospitals pathology agreed with the diagnosis of lung adenocarcinoma. The patient received Gefitinib Tablets treatment $250 \mathrm{mg}$ per day for 18 months during the perioperative period. We required the patient to return to the hospital every 6 months for CT scans of the lung and abdomen, ultrasound scans of the abdomen, and tests for blood tumour markers. Followed up to 18 months after thoracoscopic wedge-shaped partial pneumonectomy, there have two lump about $1.2 \mathrm{~cm}$ and $0.7 \mathrm{~cm}$ in diameter in upper lobe and lower lobe of the right lung by contrast-enhanced CT images(Fig. 7). According to the examination results, it was suggested that the metastasis of inferior vena cava sarcoma or the recurrence of lung adenocarcinoma, and thoracoscopic resection was decided after the discussion by thoracic surgery department. The immunohistochemical results of postoperative specimens were consistent with the metastasis of inferior vena cava sarcoma(Fig. 8).

Immunohistochemical staining showed that SMA, desmin, vimentin and h-caldesmon and Ki-67 showed $70 \%$ were positive; CD117, S100, CD34, and Dog-1 were negative. The histopathological examination of the right lung mass and the discussion of the pathological expert group suggested that it was the metastasis of inferior vena cava sarcoma. The patient received daily $12 \mathrm{mg}$ treatment with Anlotinib Hydrochloride Capsules after the last operation. Regular outpatient follow-up showed that there was no obvious recurrence and metastasis as of April 2021 (6 months after operation).

\section{Discussion And Conclusions}

Leiomyosarcomas, which are characterized by smooth muscle differentiation, can be found throughout the body where there is a vein [6]. No more than $5 \%$ of leiomyosarcomas originate from large blood 
vessels, and most of these originate from the IVC [7]. Leiomyosarcoma of the IVC was first described in 1871 [8]. According to its location, the tumour is divided into three groups: segment I: infrarenal; segment II; inter-renal and supra-renal, up to but not including the main supra-hepatic veins; and segment III: suprahepatic, up to the right atrium $[9,10]$. Because of the different dimensions, growth patterns, and locations of the tumour, leiomyosarcoma of the IVC has different symptoms. Patients present with right lower quadrant pain, back or flank pain, and leg oedema when the tumour is located in the infrarenal region, while renal involvement results in renal vein thrombosis, nephrotic syndrome or arterial hypertension. Patients with tumours in the upper portion of the IVC present with weight loss, nausea, Budd-Chiari syndrome or lower extremity oedema $[9,11]$.

Currently, a number of non-invasive imaging methods, including ultrasound, CT, and MRI, are available for the diagnosis of leiomyosarcoma of the IVC [12-15]. However, most findings from these techniques are considered to be nondiagnostic because of the nonspecific performance. Due to the retroperitoneal location, the presentation of leiomyosarcoma of the IVC is usually insidious, and most cases in the published literature are diagnosed at a late stage. The final diagnosis of leiomyosarcoma of the IVC is based on pathology.

For leiomyosarcoma of the IVC, complete surgical resection with free margins is believed to be the best modality for curative treatment $[13,16]$. The surgical approach differs depending on the tumour invasiveness and location, and the approaches include simple tumour excision with IVC repair and tumour excision with graft replacement. The efficacy of chemotherapy and radiotherapy is limited [17]. The prognosis of leiomyosarcoma of the IVC depends on the tumour size and location as well as complete surgical resection [18]. The five-year survival rate following surgery has been reported to be approximately $50 \%[9,19]$. Most leiomyosarcomas show a highly aggressive growth pattern with a high incidence of recurrence and metastasis. A study showed that tumour recurrence occurs in more than half of these patients despite complete resection [9].

In this case, pulmonary adenocarcinoma was discovered during the treatment for leiomyosarcoma of the IVC, without any symptoms. Histologically, leiomyosarcoma is characterized by a proliferation of spindle cells, with elongated nuclei and eosinophilic cytoplasm [20]. Spindle cell formation has sometimes been shown in other tumours, such as pulmonary blastoma, carcinosarcoma and mesothelioma [21, 22]. They are made of a variant of mesenchymal, epithelial, adenomatous or squamous components [23]. What is the relationship between the two tumours? According to Drlicek et al [24], there are four possibilities: a) two different primary tumours, adenocarcinoma of the lung and leiomyosarcoma of the IVC, as in the study by Takemoto et al, who reported one case with two primary tumours: leiomyosarcoma of the small intestine and pulmonary adenocarcinoma; b) adenocarcinoma with a spindle cell component; $c$ ) biphasic mesothelioma; and d) adenosquamous carcinoma of the lung with a spindle-differentiated squamous component. Fukuda et al reported a case of pulmonary adenocarcinoma with sarcomatous elements initially manifested as a submucosal tumour of the stomach. However, autopsy revealed spindle cell variants of pulmonary adenocarcinoma [25]. An immunohistochemical study and the presence of a transitional zone are important for the diagnosis of primary or metastatic tumours. In this case, the 
immunohistochemical study of leiomyosarcoma of the IVC showed that SMA myoglobin, desmin, EMA, TLE-1 and h-caldesmon were positive; Ki- 67 was $40 \%$ positive, vimentin and CD 99 were focally positive; and CD 117, CKp, CK7, Bcl-2, S100, CD31, CD34, Dog-1 and STAT-6 were negative. SMA, myoglobin, and vimentin were positive for smooth muscle differentiation, and CD 117, Bcl-2, S100, CD31, CD34, Dog-1 and CK7 were negative for non-myogenic differentiation [26]. The lung tumour showed CK7, napsin A and TTF-1 was positive. CK7, napsin A and TTF-1 have high specificity and sensitivity in the diagnosis of lung adenocarcinoma [27]. In addition, in this case, the zone of transition from adenomatous to spindle cells could not be detected. Spindle cell tumours of the IVC were not derived from pulmonary adenocarcinoma. Thus, they could be two different types of primary tumours.

Leiomyosarcoma of the IVC accompanied by lung adenocarcinoma is a rare diagnosis. We did not find any similar cases in the literature. The patient underwent surgical resection, without chemotherapy or radiation therapy. There was no recurrence or metastasis after 18 months. Therefore, surgery is still an effective treatment for patients with IVC leiomyosarcoma and lung adenocarcinoma at present. New methods of cancer diagnosis and treatment are needed to improve the prognosis of patients with IVC leiomyosarcoma and lung adenocarcinoma.

\section{Abbreviations}

IVC

inferior vena cava; CT:computed tomography; MRI:magnetic resonance imaging; SMA:smooth muscle actin; EMA:Epithelial Membrane Antigen; TLE-1:Transducin-Like Enhancer of split.

\section{Declarations}

\section{Ethics approval and consent to participate}

This study is approved by the Ethics Committee of Lanzhou University Second Hospital.

\section{Consent for publication}

We confirm that manuscript containing any individual person's data received the consent of patients and their families for publication.

\section{Availability of Data and Materials}

All data generated or analysed during this case are included in this published article.

\section{Competing interests}

No conflict of interest exits in the submission of this manuscript.

\section{Funding}


This study was supported by the Cuiying Scientific and Technological Innovation Program of Lanzhou University Second Hospital (CY2018-MS14).

\section{Authors' contributions}

Guo and Wei developed the idea of the case report, and draft this manuscript. Guo, Liu and Wang contributed to the acquisition and interpretation of data. Zhang provided critical review and substantially revised the manuscript. All authors read and approved the final manuscript.

\section{Acknowledgements}

We confirm that all authors have contributed to and agreed on the content of the manuscript. The manuscript has not been published previously, in any language, in whole or in part, and is not currently under consideration elsewhere.

\section{References}

1. Fujita S, Takahashi H, Kanzaki Y, et al. Primary Leiomyosarcoma in the Inferior Vena Cava Extended to the Right Atrium: A Case Report and Review of the Literature [published correction appears in Case Rep Oncol. 2017 Feb 2;10 (1):153]. Case Rep Oncol. 2016;9(3):599-609.

2. Alkhalili E, Greenbaum A, Langsfeld M, et al. Leiomyosarcoma of the Inferior Vena Cava: A Case Series and Review of the Literature. Ann Vasc Surg. 2016;33:245-51.

3. Mastoraki A, Leotsakos G, Mastoraki S, et al. Challenging diagnostic and therapeutic modalities for leiomyosarcoma of inferior vena cava. Int J Surg. 2015;13:92-5.

4. Kapoor R, Bansal A, Sharma SC. Leiomyosarcoma of inferior vena cava: Case series of four patients. J Cancer Res Ther. 2015;11(3):650.

5. Uramoto H. Current Topics on Salvage Thoracic Surgery in Patients with Primary Lung Cancer. Ann Thorac Cardiovasc Surg. 2016;22(2):65-8.

6. Doyle LA. Sarcoma classification: an update based on the 2013 World Health Organization Classification of Tumors of Soft Tissue and Bone. Cancer. 2014;120(12):1763-74.

7. Mertens F, ed. World Health Organization Classification of Tumours. Pathology and Genetics of Tumours of Soft Tissue and Bone. Lyon, France:IARC, 2002.

8. Sulpice L, Rayar M, Levi Sandri GB, et al. Leiomyosarcoma of the inferior vena cava. J Visc Surg. 2016;153(3):161-5.

9. Mingoli A, Cavallaro A, Sapienza P, Di Marzo L, Feldhaus RJ, Cavallari N. International registry of inferior vena cava leiomyosarcoma: analysis of a world series on 218 patients. Anticancer Res. 1996;16(5B):3201-5.

10. Kieffer E, Alaoui M, Piette JC, Cacoub P, Chiche L. Leiomyosarcoma of the inferior vena cava: experience in 22 cases. Ann Surg. 2006;244(2):289-95. 
11. Mu D, Wang D, Zhou K, Zhu B. Radiographic features of intraluminal leiomyosarcoma of the inferior vena cava: an atypical case report. Abdom Imaging. 2011;36(5):586-9.

12. Webb EM, Wang ZJ, Westphalen AC, Nakakura EK, Coakley FV, Yeh BM. Can CT features differentiate between inferior vena cava leiomyosarcomas and primary retroperitoneal masses? AJR Am J Roentgenol. 2013;200(1):205-9.

13. Ganeshalingam S, Rajeswaran G, Jones RL, Thway K, Moskovic E. Leiomyosarcomas of the inferior vena cava: diagnostic features on cross-sectional imaging. Clin Radiol. 2011;66(1):50-6.

14. Rajiah P, Sinha R, Cuevas C, Dubinsky TJ, Bush WH Jr, Kolokythas O. Imaging of uncommon retroperitoneal masses. Radiographics. 2011;31(4):949-76.

15. Hartman DS, Hayes WS, Choyke PL, Tibbetts GP. From the archives of the AFIP. Leiomyosarcoma of the retroperitoneum and inferior vena cava: radiologic-pathologic correlation. Radiographics. 1992;12(6):1203-20.

16. Illuminati G, Calio' FG, D'Urso A, Giacobbi D, Papaspyropoulos V, Ceccanei G. Prosthetic replacement of the infrahepatic inferior vena cava for leiomyosarcoma. Arch Surg. 2006;141(9):919-24.

17. Sonoda H, Minamimura K, Endo Y, et al. Complete Surgical Resection of a Leiomyosarcoma Arising from the Inferior Vena Cava. Case Rep Med. 2015;2015:342148.

18. Laskin WB, Fanburg-Smith JC, Burke AP, Kraszewska E, Fetsch JF, Miettinen M. Leiomyosarcoma of the inferior vena cava: clinicopathologic study of 40 cases. Am J Surg Pathol. 2010;34(6):873-81.

19. Hines OJ, Nelson S, Quinones-Baldrich WJ, Eilber FR. Leiomyosarcoma of the inferior vena cava: prognosis and comparison with leiomyosarcoma of other anatomic sites. Cancer. 1999;85(5):107783.

20. PHELAN JT, SHERER W. MESA P. Malignant smoothmuscle tumors (leiomyosarcomas) of soft-tissue origin. N Engl J Med. 1962;266:1027-30.

21. Kusumoto H, Takahashi I, Yoshida M, et al. Primary malignant tumors of the small intestine: analysis of 40 Japanese patients. J Surg Oncol. 1992;50(3):139-43.

22. Types histologiques des tumeurs du poumon. Deuxième édition, 1981 [Histological typing of lung tumors. Second edition, 1981 (author's transl)]. Ann Pathol. 1981;1(3):177-191.

23. ousem SA, Wick MR, Randhawa P, Manivel JC. Pulmonary blastoma. An immunohistochemical analysis with comparison with fetal lung in its pseudoglandular stage. Am J Clin Pathol. 1990;93(2):167-75.

24. Drlicek M, Liszka U, Machacek E, Grisold W, Lintner F. Spindle cell variant of pulmonary adenocarcinoma. Pathol Res Pract. 1993;189(5):586-93.

25. Fukuda T, Ohnishi Y, Katagiri J, Ohnuki K, Tachikawa S. A case of pulmonary adenocarcinoma with sarcomatous elements initially manifested as a submucosal tumor of the stomach. Acta Pathol Jpn. 1992;42(6):454-9.

26. Li X, Liu HG, Xie XJ, Han YD, Li M. Clinicopathologic study of sinonasal teratocarcinosarcoma and its contrast with olfactory neuroblastoma. Zhonghua Bing Li Xue Za Zhi. 2008;37(7):458-64. 
27. Hutchinson BD, Shroff GS, Truong MT, Ko JP. Spectrum of Lung Adenocarcinoma. Semin Ultrasound CT MR. 2019;40(3):255-64.

\section{Figures}

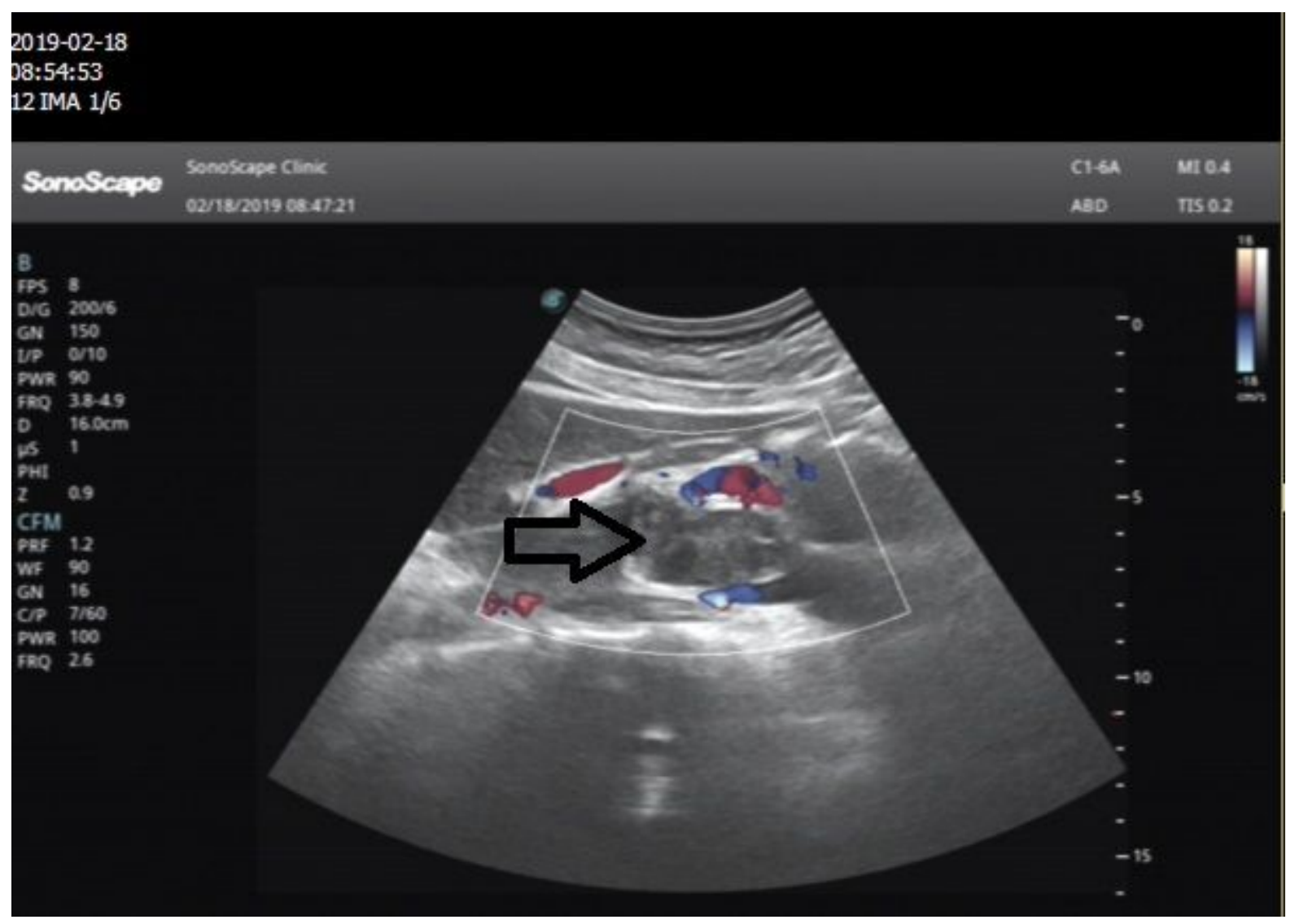

\section{Figure 1}

Abdominal ultrasonography images in the epigastric sagittal view: a hypoechoic, irregularly shaped solid mass was seen adjacent to the inferior vena cava (IVC). 

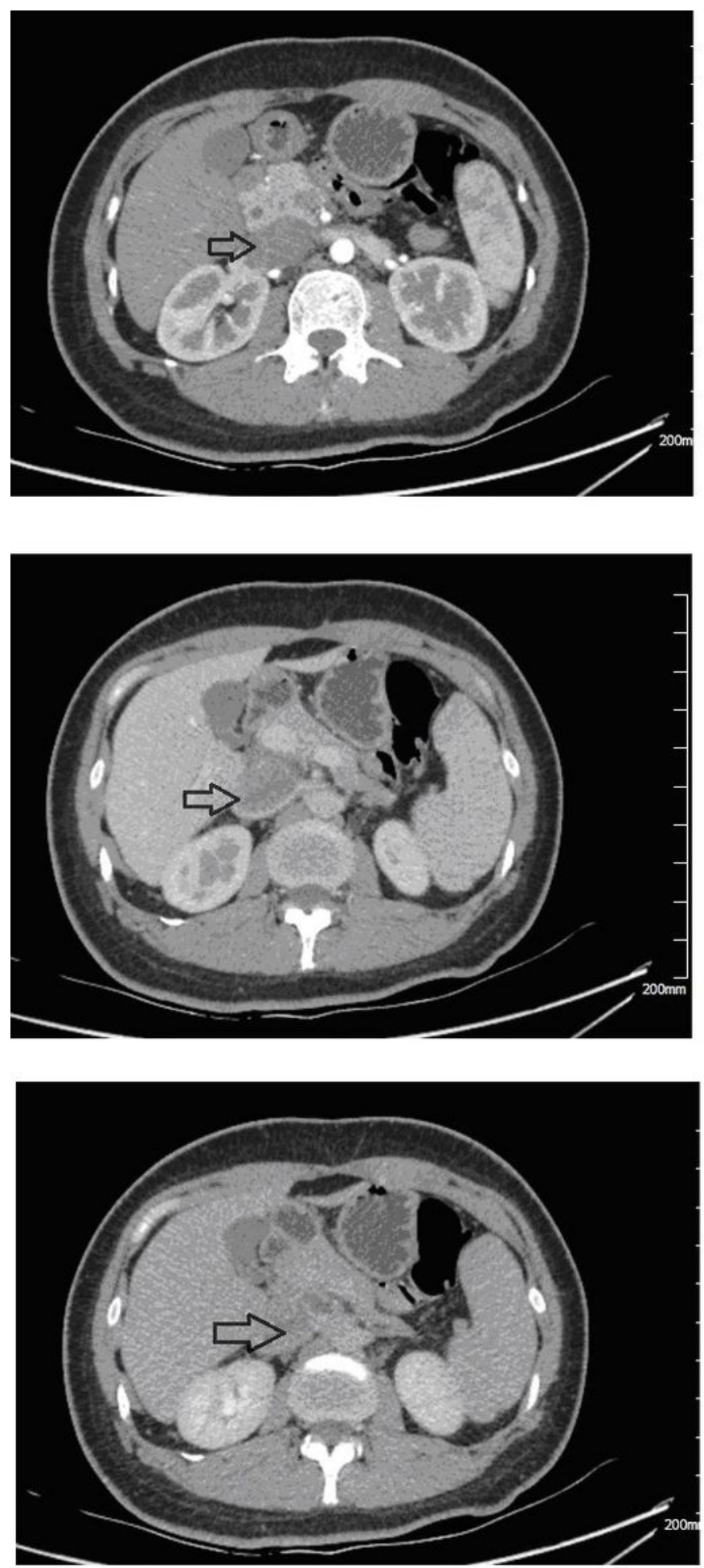

Figure 2

Preoperative contrast-enhanced CT images. A ball-shaped mass was revealed adjacent to the IVC and superior to the right renal vein at the arterial phase, and the border between the tumour and IVC was indistinct. Preoperative contrast-enhanced CT images. The border between the tumour and IVC was indistinct in the venous phase. Preoperative contrast-enhanced CT images. The border between the tumour and IVC was indistinct in the delayed phase. 


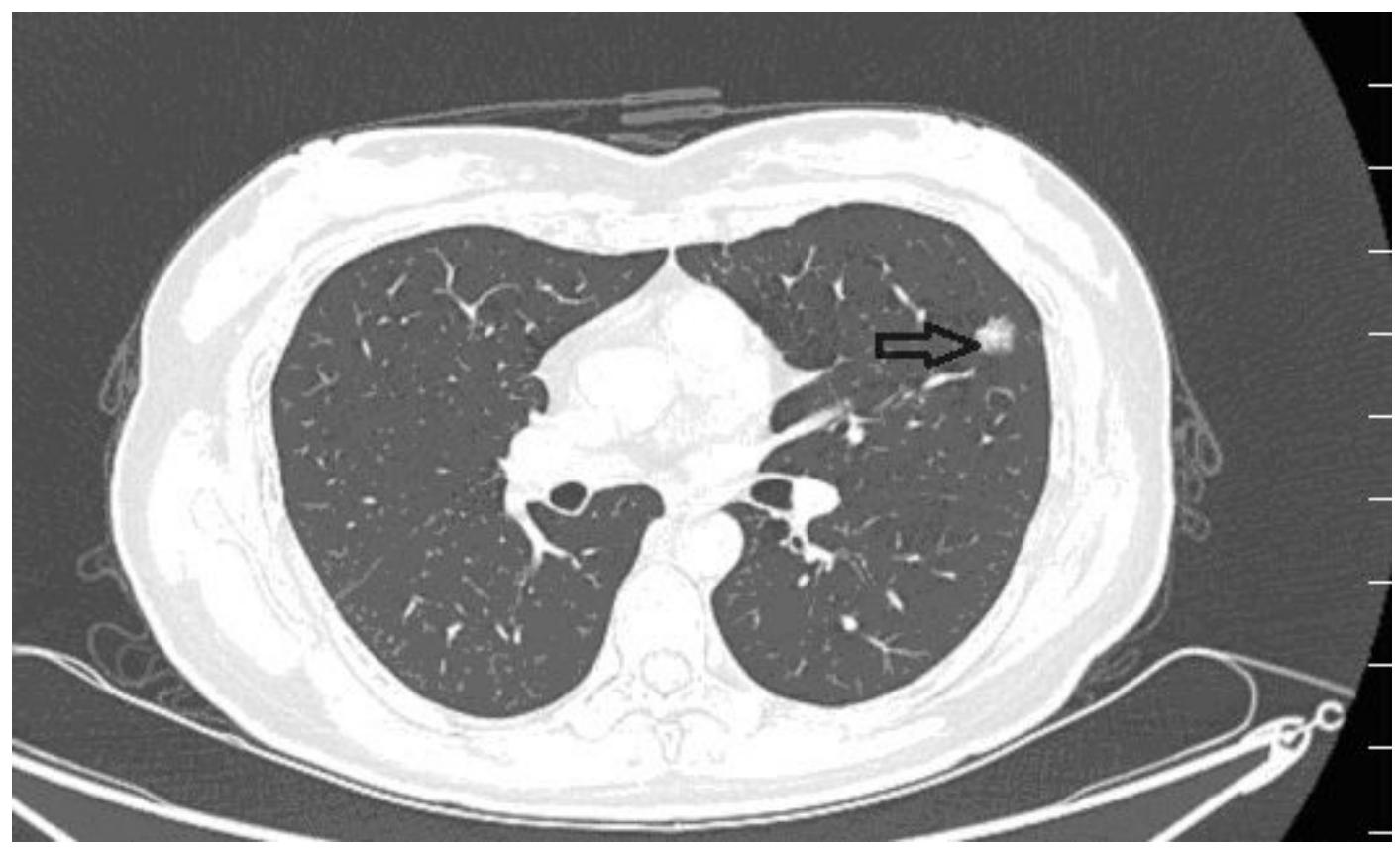

Figure 3

Preoperative contrast-enhanced CT images revealed a ball-shaped mass with heterogeneous enhancement in the left lung lobe.

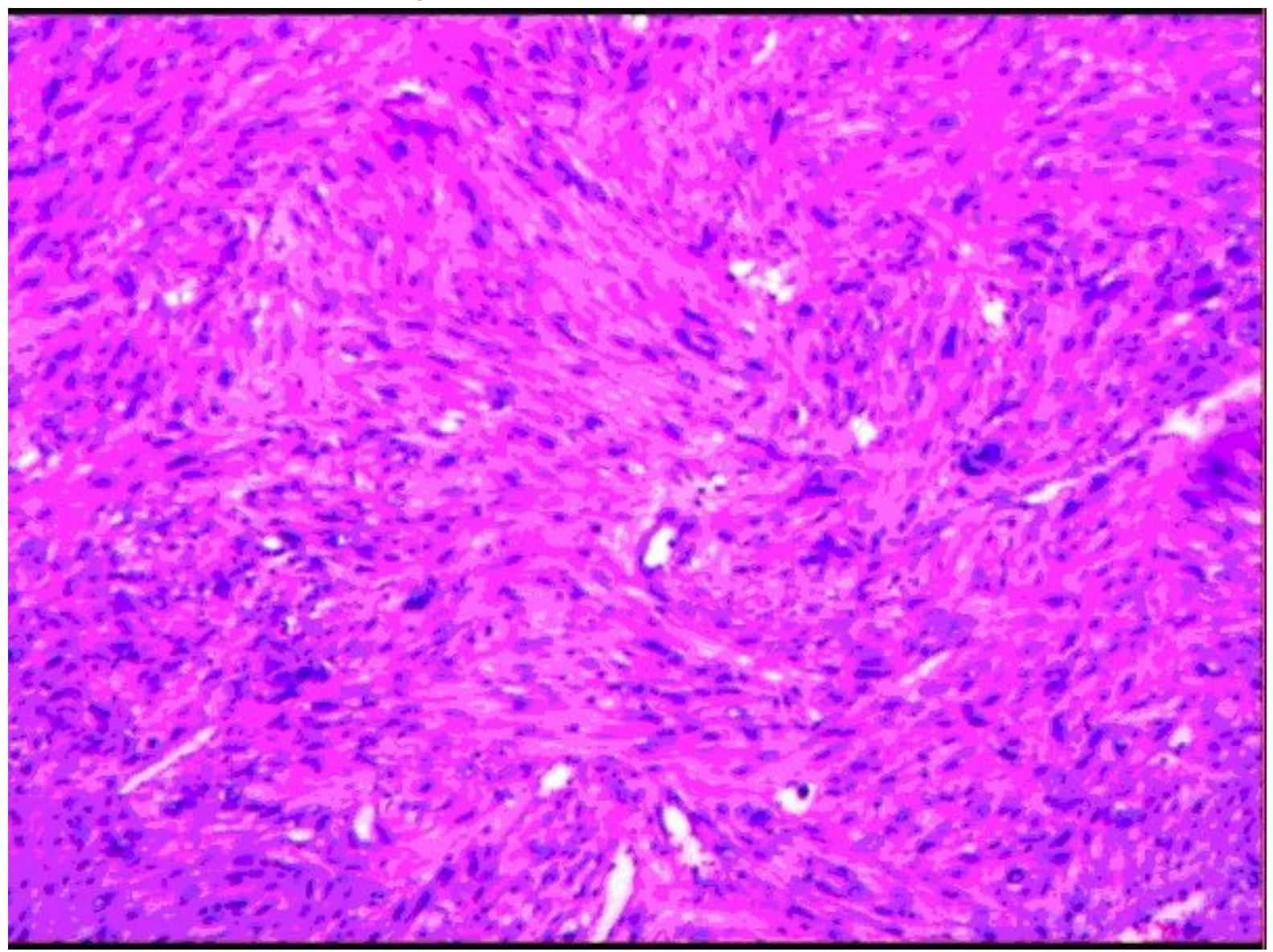

Figure 4 
Image showing spindle-shaped tumour cells with areas of hypercellularity that were arranged in fascicles, bundles and interlacing patterns.

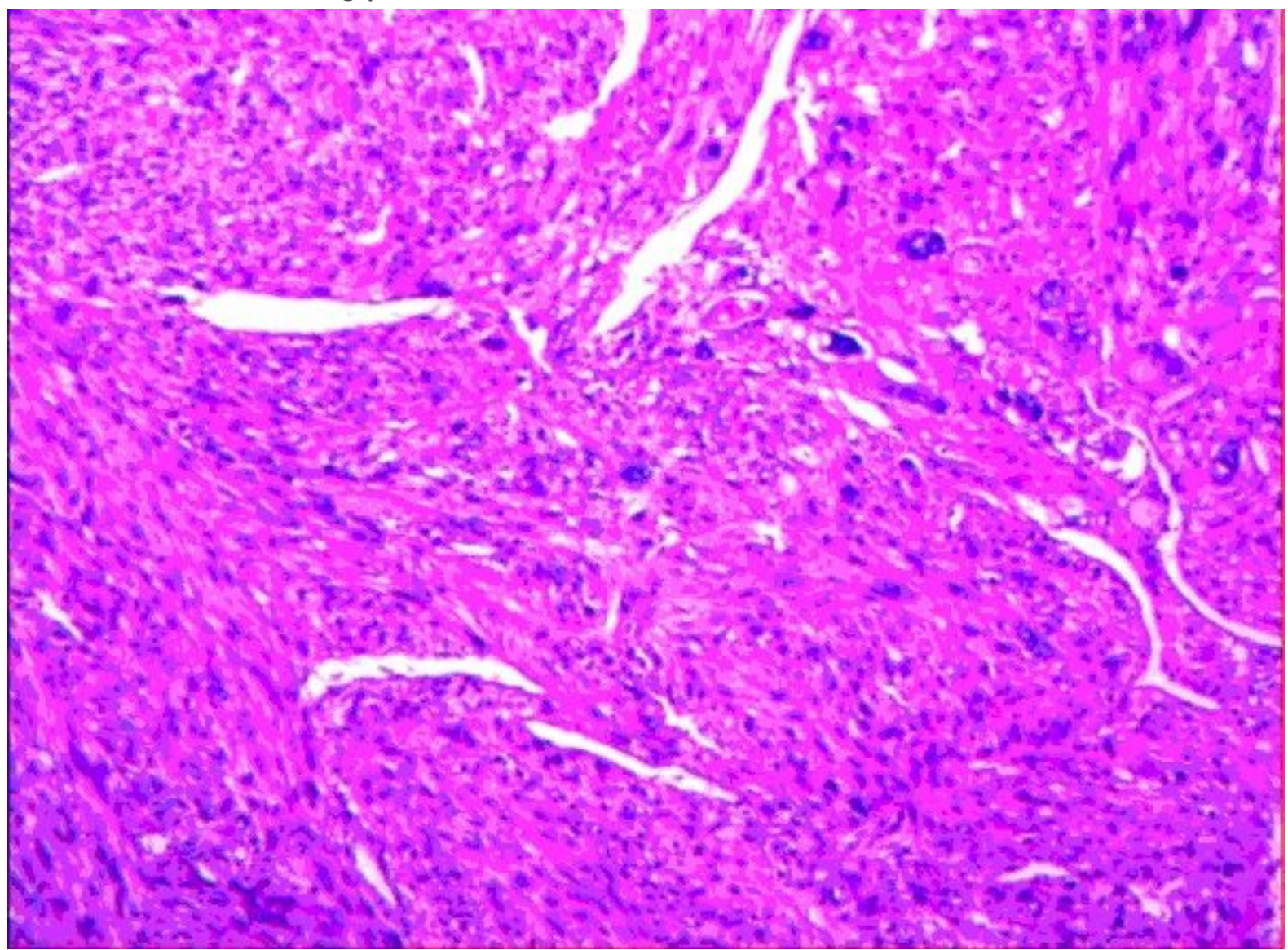

\section{Figure 5}

The nuclei were hyperchromatic, blunt-ended, and centrally located and had some degree of atypia and pleomorphism. A few fused nuclei were also seen. 


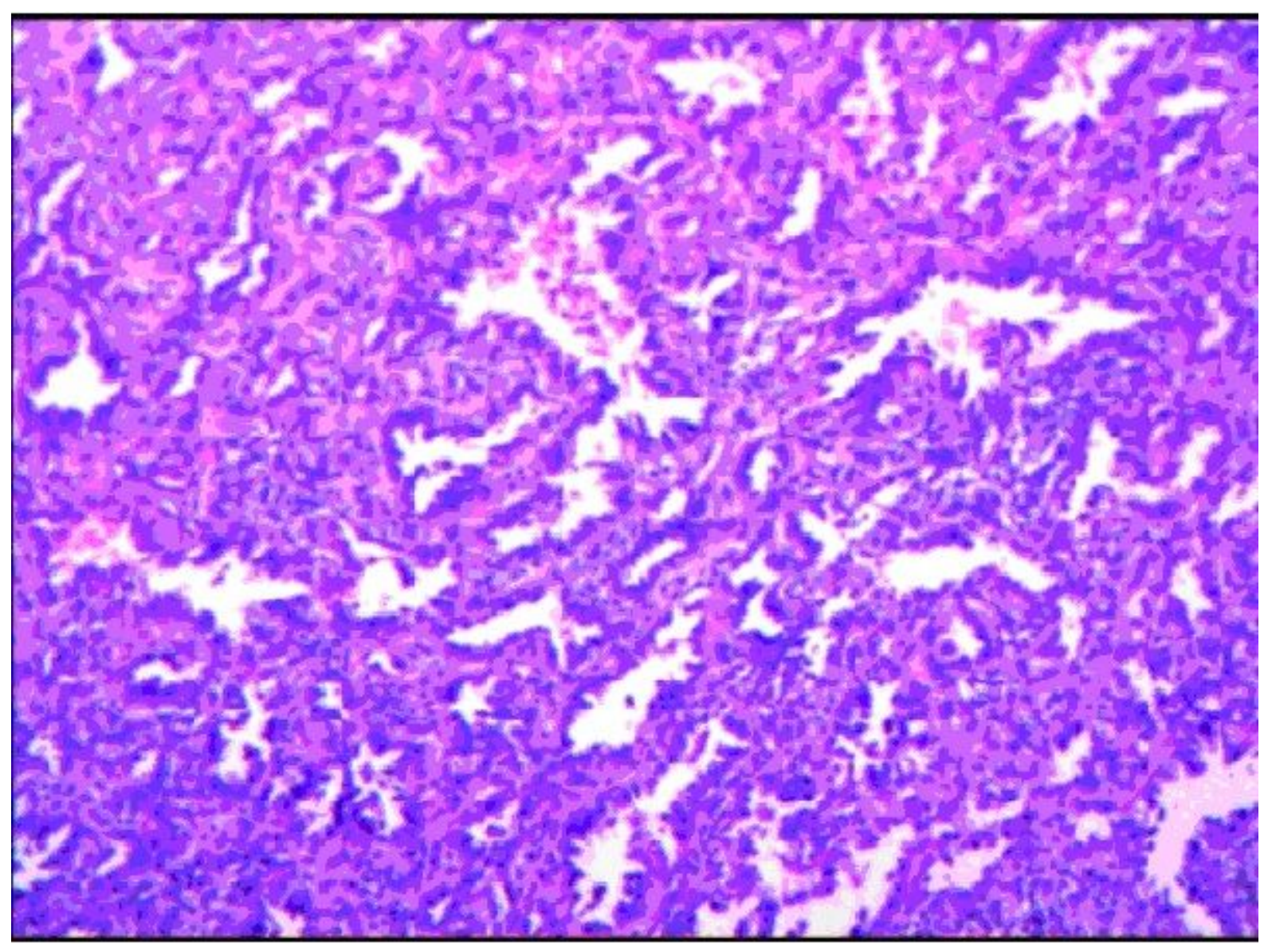

\section{Figure 6}

The histopathological examination of the excised mass combined with the pathology expert group discussion considered lung adenocarcinoma. 

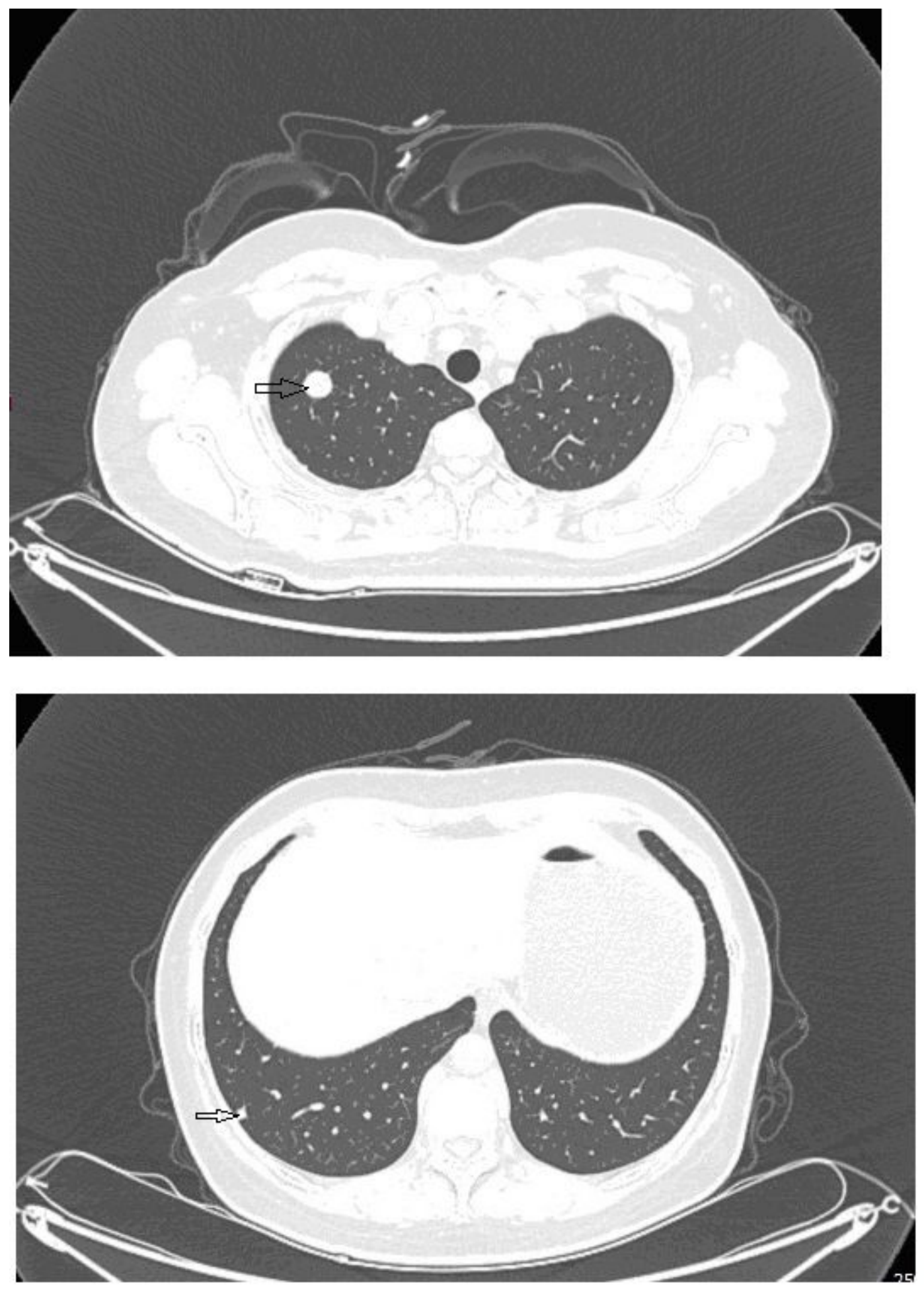

Figure 7

Contrast-enhanced CT images at 18 months after second surgery: lump in upper lobe of the right lung. Contrast-enhanced CT images at 18 months after second surgery: lump in lower lobe of the right lung. 

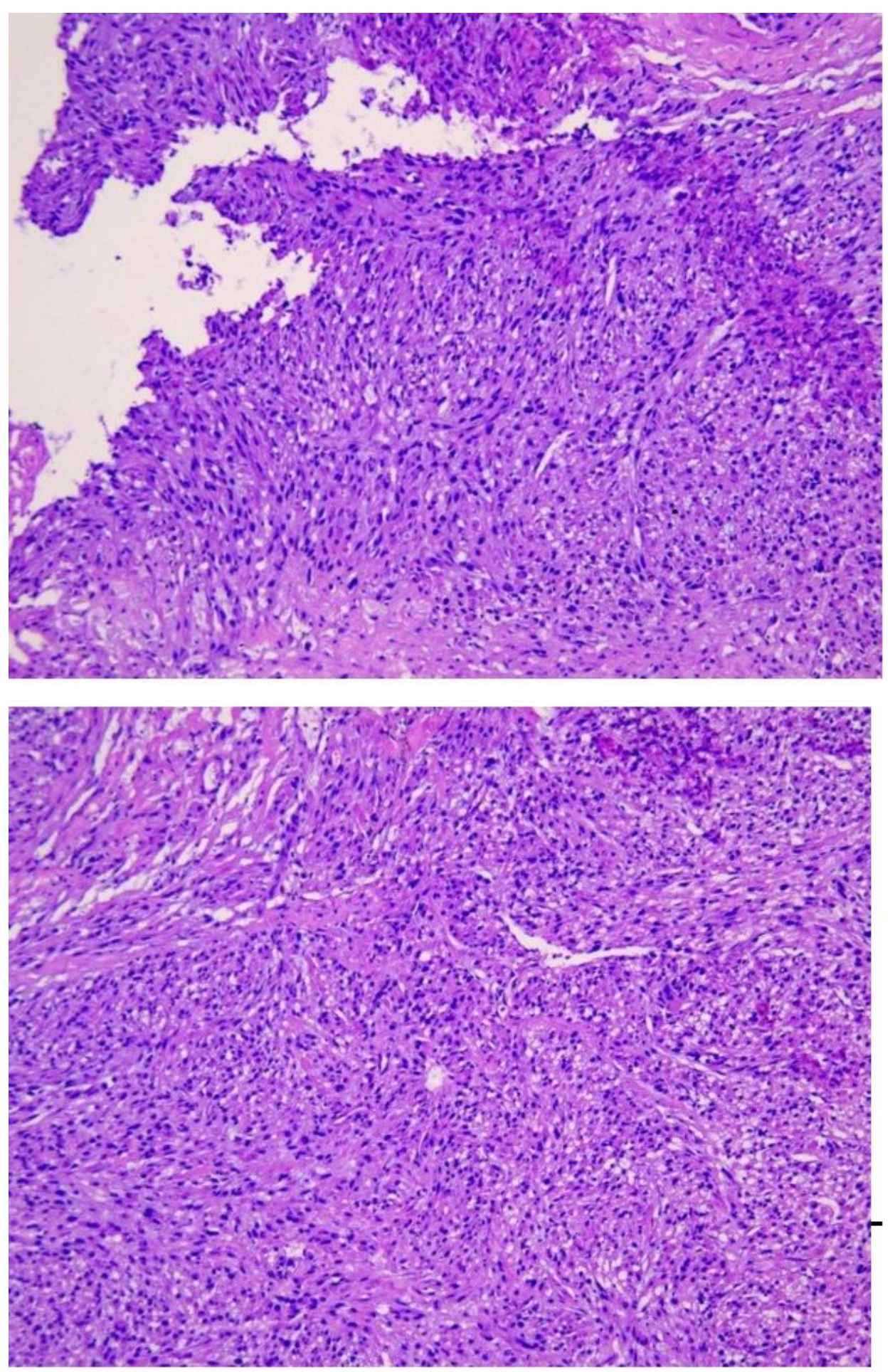

\section{Figure 8}

The metastasis of inferior vena cava sarcoma in upper lobe of the right lung. The metastasis of inferior vena cava sarcoma in lower lobe of the right lung. 